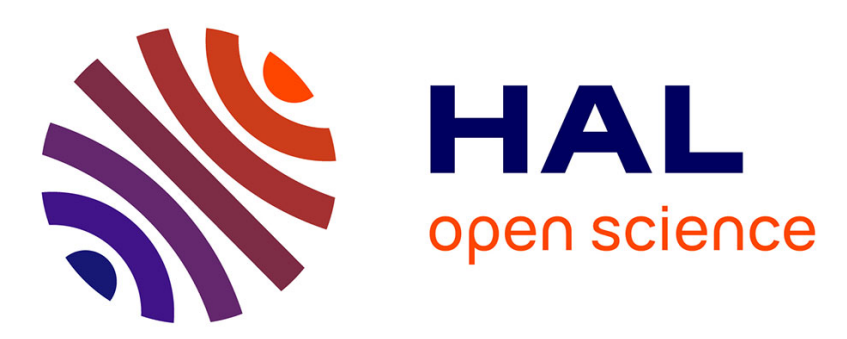

\title{
Quantification strategies for MRI
}

Olivier M Girard, Ludovic de Rochefort, Marie Poirier-Quinot, Luc Darrasse, Robert F Mattrey

\section{To cite this version:}

Olivier M Girard, Ludovic de Rochefort, Marie Poirier-Quinot, Luc Darrasse, Robert F Mattrey. Quantification strategies for MRI. Molecular Imaging Techniques: New Frontiers, 2013, MRI - recent advances and new horizons, 10.4155/EBO.13.146 . hal-02269984

\section{HAL Id: hal-02269984 https://hal.science/hal-02269984}

Submitted on 19 Jan 2022

HAL is a multi-disciplinary open access archive for the deposit and dissemination of scientific research documents, whether they are published or not. The documents may come from teaching and research institutions in France or abroad, or from public or private research centers.
L'archive ouverte pluridisciplinaire HAL, est destinée au dépôt et à la diffusion de documents scientifiques de niveau recherche, publiés ou non, émanant des établissements d'enseignement et de recherche français ou étrangers, des laboratoires publics ou privés. 


\section{Molecular imaging techniques-new frontiers}

\section{Chapter 9: Quantification strategies for MRI}

Olivier M. Girard ${ }^{1,2}$, Ludovic de Rochefort ${ }^{3}$, Marie Poirier-Quinot ${ }^{3}$, Luc Darrasse ${ }^{3}$, Robert F. Mattrey ${ }^{2}$

1/ Centre de Résonance Magnétique Biologique et Médicale, CRMBM - UMR 7339, CNRS / Aix Marseille Université, Marseille, France

2/ Department of Radiology, University of California San Diego, San Diego, California, USA

3/ Imagerie par Résonance Magnétique Médicale et Multi-Modalités, IR4M - UMR 8081, CNRS / Université Paris - Sud 11, Orsay, France

\section{Corresponding author:}

Olivier M. Girard

Centre de Résonance Magnétique Biologique et Médicale, CRMBM - UMR 7339, CNRS / Aix - Marseille Université,

Faculté de Médecine, 27 Boulevard Jean Moulin, 13385 Marseille Cedex 05

Phone: :+33 659703509

Email: olivier.girard@univ-amu.fr and olivier.gigi@gmail.com

\section{Abstract:}

Quantification strategies have been a long standing challenge since the early days of MR molecular and cellular imaging. Quantitative imaging is desirable in the framework of comparative or follow-up studies in order to establish objective evidences of a specific target expression pertaining to a specific disease. Numerous strategies of increasing complexity have been proposed to address this need. In this chapter, we briefly review common strategies related to quantitative molecular imaging for MRI and we discuss their respective outcomes. We focus on relaxation Contrast Agents (CAs) that generate $\mathrm{MR}$ contrast by affecting the proton relaxation times, and which are the most common kind of CAs used in MRI. Particular attention is paid on cutting edge applications enabling local CA quantification as well as distinction of the bound/activated sub-population of the CA. 


\section{Introduction}

Quantification strategies have been a long standing challenge since the early days of MR molecular and cellular imaging. Quantitative imaging may be required to establish the in vivo proofof-principle of a new compound efficiency, and it is desirable in the framework of comparative or follow-up studies performed on multiple patients. Typical molecular imaging studies, for instance, consist in comparing the bio-distribution of a targeted contrast agent (CA) with its non-targeted counterpart. Such assessment has to be repeated on several patients in order to overcome the inherent biological variability and to extract statistically significant results, ultimately establishing objective evidences of a specific target expression pertaining to a specific disease. Hence, numerous quantitative strategies of increasing complexity have been proposed to address this need and perform such studies. In this chapter, we briefly review common strategies related to quantitative molecular imaging for MRI and we discuss their respective outcomes. Particular attention is paid on the most cutting edge applications enabling local CA quantification as well as distinction of the bound/activated sub-population of the CA.

Simple quantification strategies consist is measuring the fractional signal change (i.e. \% signal enhancement/drop) due to the presence of the CA, on a given MR image. The total area (or volume) of signal enhancement may be easily calculated as well to deliver some quantitative index of the CA distribution. This can be done either using a well chosen reference intensity tissue or by using registered pre- and post- injection images. Although popular, these strategies are not really quantitative but should rather be considered as pseudo-quantitative, since they do not provide quantitative information on the CA or the target distribution themselves, but instead dilute the relevant local information in a high-level global parameter. In addition, such measurements are prone to multiple biases, especially the choice of the MR sequence parameters used to acquire the weighted image, which makes multi-center and cross-vendor studies difficult to perform. Conversely, more complex data processing procedures may be used to estimate parametric maps (e.g. relaxation time maps) which provide, at least in theory, sequence-independent parameters that can be compared from site to site. The underlying interpretation in terms of CA concentration may not be univocal, but at least the data are truly quantitative and can be compared from one study to another.

Actual CA quantification is certainly the ultimate goal of quantitative imaging protocol applied to CA detection. Indeed, knowledge of the CA concentration is a prerequisite to derive the number of targeted receptors or labeled cells as well as the quantity of carried drug when using theranostic agents for instance, which is ultimately the relevant information typically expected from molecular imaging studies. Robust CA quantification is still very challenging for targeted CA such as those used in molecular imaging applications, mostly because the CA coexists in multiple states (i.e. free and targeted form) at the same time in the sample and these states do typically have different MR contrast efficiency. Current advanced methods hold promise for absolute CA quantification, including the specific assessment of the targeted population of a compound only.

In the following we focus on relaxation CAs, as opposed to chemical shift reagents (e.g. CEST imaging agents) or multinuclear reagent (e.g. ${ }^{19} \mathrm{~F}$ probes), i.e. we focus on CAs that generate MR contrast by affecting the proton relaxation times, which are the most common kind of CA used in MRI. 


\section{$M R$ is an indirect detection modality}

As opposed to nuclear medicine modalities, usual CAs cannot be observed directly using MRI because they do not provide intrinsic MR signal and this adds an extra level of complexity to the detected signal (see Chapter 3 for details on direct detection using $\mathrm{X}$-nuclei, i.e. non ${ }^{1} \mathrm{H}$ ). Instead, CA detection is based on their interaction with surrounding protons through several mechanisms affecting relaxation rates and resonance frequency. This indirect nature of CA detection makes it prone to multiple biases that need to be accounted for to quantify the amount of CA directly by imaging.

MRI detects CA through their effect on mobile water protons. Relaxation MR contrast agents shorten proton relaxation times through magnetic interaction with neighboring protons and subsequent exchange of these protons to the bulk water, which amplifies and propagates the CA effect. In general, the MRI signal is proportional to the local proton density and is weighted by some function of the longitudinal $\left(T_{1}\right)$ and transverse $\left(T_{2} / T_{2}{ }^{*}\right)$ relaxation times of the tissues - the actual weighting formulae depends on the actual pulse sequence that is used to capture the image. The effect of MR CA is usually summarized in term of their efficiency to speed up proton relaxation processes - defined as relaxivity, according to the following formulae:

$$
R_{i}=R_{i 0}+r_{i}[C A] \text {, with } i=1,2 \text { or } 2^{*} \text {, Eq. } 1
$$

where $R_{i}=1 / T_{i}$ is the relaxation rate observed in presence of $C A, R_{i 0}$ is the baseline tissue relaxation rate, $r_{i}$ is the CA relaxivity and $[C A]$ its concentration. This relation may apply to both relaxation mechanisms, i.e. longitudinal $T_{1}$ and transverse, $T_{2}$ and $T_{2}{ }^{*}$. By acquiring appropriate weighted images of a given sample it is thus possible to visualize CA by MRI, although the measured MR signal is a complex non-linear function of the CA content.

While usual MRI contrast agents affect both proton relaxation times (longitudinal and transverse), they are typically classified according to their dominant mechanism:

1/ Paramagnetic compounds such as Gadolinium (Gd) chelates [1] have a $r_{1} / r_{2}$ ratio close to 1 , thus they mainly affect $T_{1}$ (because $R_{10}<R_{20}$ for most tissues) and hence provide positive contrast on $T_{1}$ weighted images.

2/ Superparamagnetic compounds such as iron oxide nanoparticles [2] (IONPs) have a low $r_{1} / r_{2}$ ratio (i.e. $<<1$ ), thus they usually have dominant $T_{2}$ effect, leading to signal voids (negative contrast) on $T_{2^{-}}$ or $\mathrm{T}_{2}{ }^{*}$ - weighted scans.

Although this simplified classification does not account for all encountered effects and observed contrast types it certainly corresponds to the most popular use for either type of compounds. As alternative examples, Gd-based CAs are routinely used for bolus tracking studies to measure perfusion (DSC-MRI for dynamic susceptibility contrast); a technique that relies on the negative $\mathrm{T}_{2}$ * effect arising around vessels containing high concentrations of $\mathrm{Gd}$. Conversely, recent advances in MR pulse sequence methodology have enabled probing the $T_{1}$-effect of IONPs using utrashort echo time (UTE) scans, hence providing positive contrast detection of such compounds (see Girard et al. [3] for a general theory of CA-induced MR contrast and a detailed comparison of the $T_{1}$ and $T_{2}$ effects of IONPs). 


\section{Parametric map estimation}

To address CA quantification challenge, multiple weighted images can be used to extract parametric maps which are quantitative maps of a parameter of interest such as $T_{1}$ or $T_{2}$ (i.e. images that scale directly proportional with the corresponding parameter). These maps are usually obtained from several MR images acquired with varying imaging parameter, and computed using least-square data fitting algorithms based on analytical MR signal description. For instance, multiple-echo spinecho or gradient-echo sequences allow for $T_{2}$ and $T_{2}{ }^{*}$ quantification, respectively, using an exponential decay model. Similarly, inversion recovery prepared sequences acquired using various inversion times allow for $\mathrm{T}_{1}$ quantification, using an exponential recovery model.

Relaxation time quantification has been a very popular way to extract information about CA content. Indeed, using a relaxation time map and Eq.1 it is possible to derive a CA concentration map, assuming that the baseline tissue relaxation time is known or has been measured prior to CA injection, and assuming a constant value for the corresponding relaxivity as well. As we will see later, this last assumption is a critical issue related to CA quantification. Relaxation time quantification has been applied for years in the MR community to study $C A$, and it is actually the method of choice to measure CA relaxivity, as Eq.1 may also be used to infer relaxivity using in vitro samples of known CA concentration.

Despite its apparent simplicity, relaxation mapping methods have been the focal point of many researchers for years until now, as it is still challenging to get robust and accurate relaxation time measurements in a short scan time. Multiple confounding factors may affect the measured signal and refined signal models have to be considered. RF magnetic field inhomogeneities, for instance, need to be corrected to get reliable $T_{1}$ estimates [4]. In the context of $T_{2}$ * quantification, which is the most sensitive parameter to IONPs [5], significant work has been done to account for macroscopic $B_{0}$ inhomogeneities that arises close to air/tissue interfaces, and this has made possible reliable in vivo $\mathrm{T}_{2}{ }^{*}$ measurements [6].

\section{CA relaxivity modulation scenarios}

An important challenge for CA quantification based on relaxation mapping results from the indirect nature of CA detection. Unfortunately, CA relaxivity is not constant in many practical conditions, which breaks down the validity of Eq.1. Indeed, because the relaxivity depends on magnetic interactions of the CA with neighboring protons as well as water exchange and diffusion around the magnetic core, it is affected by the microscopic distribution of the compound as well as the microstructure of the tissue of interest, which obviously influence the magnetic interaction strength and the water mobility [7-9]. Thus, CA relaxivity may vary spatially and temporarily during a single experiment, as the $C A$ experiences various micro-environments from the injection site to the targeted receptor. This is especially true in the framework of molecular imaging CAs which are designed to interact with other molecules $[10,11]$ and for which amplification strategies have been intended, such as the accumulation of CA inside cells. In this later case, the modification of the CA local distribution is obtained intentionally to enhance the MR signal and hence facilitate the 
detection of small doses of the targeted receptors or cells (see Chapter 3 for general rules of detection sensitivity). However, this complicates tremendously CA quantification based on relaxation time measurements because a given relaxation time variation may be attributed either to a concentration change or to a change of contrast efficiency (i.e. relaxivity, see Eq.1).

In a global molecular imaging perspective, such MR contrast switching capabilities are actually fortunate because they have allowed CAs to probe tissue microenvironment (e.g. OFF or silent state for the native CA state, and ON or active state upon receptor binding). Thus it has been a driving force in developing responsive agents that have enabled detection of specific targeted receptor or enzymes (e.g. see following references $[10,12]$ for various responsive CA designs and principles). Illustrations of $C A$ relaxivity modulation scenarios due to receptor binding or cell labeling are presented in Fig 1. A well known effect related to paramagnetic CA is the modulation of the tumbling rate of the complex following binding with a high molecular weight target, which strongly affects the $r_{1}$ relaxivity profile (Fig. 1 a). Such effect can be exploited to generate highly specific image contrast related to the bound form of the CA only, and is known as delta relaxation enhanced MR (dreMR [13]). The dreMR technique is a promising candidate for quantification of paramagnetic CA based on $T_{1}$ relaxation measurements since it totally suppresses the non-specific (i.e. unbound) components of the CA. Hence with this technique, no more MR signal comes from the free CA component and only targeted CAs, of known relaxivity (assuming no other mechanism is modulating relaxivity), contribute to the MR signal [13]. Note however that such an approach requires some dedicated piece of hardware and is still in an early research phase (see Chapter 3 for more details).

Another well documented $T_{1}$ modulation effect is related to the slowdown of the water exchange regime that may follow CA internalization into cellular compartments (Fig. 1 b): the time needed for the water molecules to cross cellular membranes and propagate the relaxation enhancement effect to the bulk water is lengthened and this results in saturation or "quenching" of the $T_{1}$ contrast [14-16]. Although, this latter effect may be understood based on complex multiple compartments and exchange ways modeling, it is rather difficult to predict the actual relaxivity value following targeting and subsequent cell internalization, hence hindering quantification.

For cellular imaging using IONPs, a third relaxation modulation effect is related to the $T_{2}$ and $\mathrm{T}_{2}{ }^{*}$ susceptibility contrasts mechanism. As opposed to the $\mathrm{T}_{1}$ effect which relies on close water access to the magnetic core of the CA, the susceptibility contrast is a long distance mechanism affecting both $T_{2}$ and $T_{2}{ }^{*}$, and which is mediated by the strong magnetic field inhomogeneities induced by the IONPs (Fig. $1 \mathrm{c}$ ). The characteristic size of the magnetic field inhomogeneities is a critical factor here and thus accumulation of multiple IONPs into clusters in the cellular space has a strong effect on induced MR contrast. For free IONPs, the random motion of water molecule leads to an irreversible loss of phase coherence because each single proton experience random magnetic field variations. In this case $T_{2}$ and $T_{2}{ }^{*}$ relaxivities have somewhat similar intensity. In contrast, for large IONP clusters the characteristic size of the field heterogeneities becomes larger than the random walk of the water molecules during the time scale of the MR experiment. Hence the induced spin dephasing is reversible using a spin echo and the associated $\mathrm{T}_{2}$ effect is strongly reduced. Conversely, the $\mathrm{T}_{2}{ }^{*}$ effect reaches its maximum intensity as described by the static dephasing regime theory (SDR) $[5,9]$. In this context $R_{2}$, defined as $R_{2}{ }^{*}-R_{2}$ (usually reported as related to "large scale" magnetic field inhomogeneities) is mostly sensitive to large IONP clusters, i.e. IONP labeled cells. 


\section{Multiparametric imaging}

Based on this concept, Kuhlpeter et al. [17], have shown that cell-internalized IONPs can be differentiated from freely distributed IONPs using joint $\mathrm{T}_{2^{-}}$and $\mathrm{T}_{2}{ }^{*}$-mapping, demonstrating the benefit of multiparametric relaxation mapping. This was confirmed recently by Girard et al. [18] using another IONP type and cell line, and pushed further to assess the full quantification potential of such a multiparametric approach. In this study, all four contrast mechanisms $\left(T_{1}, T_{2}, T_{2}{ }^{*}\right.$ and $\left.T_{2}{ }^{\prime}\right)$ as well as magnetic susceptibility were studied in vitro using cell culture samples (cf. Fig 2 ). In addition IONP content was quantified on the same samples using inductively coupled plasma - mass spectroscopy, and considered as the gold standard. From these measurements it was shown that quantitative relaxation data yield quantitative estimates of the IONP internalized fraction providing that the local particle concentration is know. In addition the $T_{2}{ }^{\prime}$ contrast mechanism was found very consistent with the SDR theory (i.e. it was predictable), allowing for cell-internalized fraction estimation without the need for preliminary calibration of the internalized relaxivity curve. Last but not least, this study demonstrated that magnetic susceptibility, as measured through IONP induced MR frequency shift, is a potential candidate for absolute CA concentration estimation since it does not depend on whether the IONPs are free or internalized into cells (Fig 3). An important limitation of this study however was that the susceptibility was not measured by imaging means (MR spectroscopy was used instead), and a well defined shape of the sample was necessary to extract susceptibility estimates from measured data. Hence direct translation of the proposed methodology to in vivo application is not possible. To overcome this limitation, alternative susceptibility quantification approaches are necessary.

\section{Quantitative Susceptibility Mapping (QSM)}

Recently, development of complex post-processing algorithms has made possible to quantify the magnetic susceptibility of tissues directly by imaging means using MRI. In contrast with its predecessor susceptibility weighted imaging (SWI) [19], which was only qualitative, a new family of techniques has emerged, called quantitative susceptibility mapping (QSM) [20,21], which delivers quantitative measurements of the magnetic susceptibility distribution. In addition to CA quantification, several other research areas may benefit from QSM since it is particularly promising for venous blood oxygen saturation level, endogenous iron as well as local myelin content estimation, and hence should yield to multiple potential clinical applications.

The QSM technique relies on the phase information of the MR signal that was often disregarded so far. Local phase data is directly proportional to the MR frequency shift, and hence can be used to detect local field inhomogeneities induced by magnetic material such as IONPs, which modulate the proton resonance frequency in their neighborhood. In short, a magnetic source distribution consistent with the observed field inhomogeneities is reconstructed by solving an inverse problem with similar concepts that found in magneto-encephalography (MEG). An important complexity, however, lies in the ill-posed inverse problem. Tremendous efforts have been carried out recently to develop dedicated algorithms able to regularize the solution by adding priors into the 
reconstruction, and this has been addressed in many different ways by several groups [20-24]. Other important challenges need to be addressed along this complex post-processing chain, especially robust phase unwrapping as well as background field variation removal, which are prerequisite steps to obtain high quality susceptibility maps.

In the context of IONPs quantification, QSM-like methods have been successfully applied in a couple recent studies [22,23], although no actual susceptibility maps were generated (or equivalent parameter such as magnetic moment map). Figure 4 illustrates the potential of QSM method to quantify IONP in a preliminary cellular imaging experiment; an actual magnetic moment map is displayed on Fig. 4. c) where the image intensity is directly proportional to IONP content. Besides, QSM has been proven efficient for quantification of Gd-based agents [25] as well. However, because of multiple confounding factors, and various different approaches presented in the literature to address them, robustness and sensitivity of the QSM technique is still an open question. In the near future it is important to reach consensus regarding which particular QSM reconstruction algorithm and associated parameters have to be used to obtain reliable results. This would definitely promote wider acceptance of QSM as a routine tool, and ultimately yield to a robust CA quantification method.

\section{Conclusion}

Molecular imaging MR contrast agents have been designed to interact with specific molecules and cellular receptors in a way that modifies their relaxation contrast efficiency. This translates into modulation of the usual relaxation mechanisms upon specific binding or activation, which has enabled specific detection of targeted-CA and has made MR a molecular imaging modality. Unfortunately, modulating CA relaxivity complicates CA quantification at the same time, because both free and activated CA pools typically coexist and hence no single relaxivity value can be used to extract CA concentration from relaxation data. Alternatively, magnetic susceptibility measurements have been shown promising for quantifying IONPs in cellular imaging studies, and are suitable for paramagnetic CA quantification as well. Recent advances in modeling and reconstruction algorithms have enabled Quantitative Susceptibility Mapping using the phase data of the complex MR signal. This technique holds great promise for robust absolute CA quantification in the future, which should open new horizons to address current issues in MR molecular imaging. 
Chapter Summary: (5-8 key sentences are pulled out as bullet points to highlight the main points of the chapter.)

When using relaxation quantification to perform molecular imaging studies, caution must be paid on the underlying interpretation in terms of CA content.

In general, multiparametric approaches should be preferred over single parameter estimation, as they provide insight into CA microdistribution and activation, e.g. targeting or cell-internalization.

Quantitative Susceptibility Mapping holds great promise for absolute CA quantification, although it is still limited to a few research centers because of complex implementation scheme.

Combining susceptibility and relaxation time mapping should provide new quantitative indexes, allowing for absolute quantification of CA content as well as the activated fraction.

\section{Key terms: (up to 4)}

Weighted images: MRI typically exhibits weighted images where the image intensity is a function of a given parameter, such as $T_{1}, T_{2}$ or the proton density. However weighted images are only qualitative and do not quantify the underlying parameter of interest (i.e. the image intensity is not proportional to the parameter of interest). The scale of a weighted image is unit-less and arbitrary.

Parametric maps: Parametric maps (e.g. T2-map or magnetic susceptibility-map) are quantitative images. Such maps have been typically reconstructed from multiple weighted images using dedicated post-processing algorithms. The scale of a parametric map is directly proportional to the corresponding parameter of interest.

Relaxivity: Relaxivity is defined as the efficiency of a given amount of CA to speed up relaxation processes of surrounding protons. It may relate to either $T_{1}$ or $T_{2} / T_{2}{ }^{*}$ contrast mechanism. Relaxivity units are $\mathrm{mM}^{-1} \cdot \mathrm{s}^{-1}$.

\section{Key learning points: (up to 4)}

1/Relaxivity depends on the CA microdistribution within a tissue, which hinders CA quantification from relaxation data.

When using relaxation quantification to perform molecular imaging studies, caution must be paid on the underlying interpretation in terms of CA content.

2/Magnetic susceptibility provides an independent measurement of local magnetic content, which is promising for robust CA quantification.

3/Combined susceptibility, $T_{2}$ and $T_{2} *$ measurements enable absolute quantification of iron oxide nanoparticles as well as cell internalized fraction in cellular imaging studies. 


\section{Legends}

Figure 1: Relaxivity modulation scenarios following receptor binding and cellular uptake. a) Tumbling rate effect following receptor binding for paramagnetic CA. Relaxation dispersion profile for $0.25 \mathrm{mM}$ of MS-325 (Gd) in pure water and in 0.6 mM HSA. See Girard et al. Proc. of ISMRM 2008, 1656, for more information. b) Relaxivity quenching effect following cell internalization and endosomal encapsulation. Such effect is due to the water exchange slowdown through multiple cellular membranes, and hence may affect all kinds of CA. Adapted with permission from Aime et al., Acc. Chem. Res. 2009, 42, 822. Copyright 2009 American Chemical Society. c) Effect of IONP clustering on the $T_{2}$ and $T_{2}{ }^{*}$ contrast regime. $R_{2}{ }^{\prime}=R_{2}{ }^{*}-R_{2}$ is mostly sensitive to IONP clusters such as those encountered into labeled cells.

Figure 2: Relaxation rates and corresponding relaxivities for various fractions of free and cellinternalized IONPs. Large relaxivity variations are observed between different groups, evidencing modulation of relaxivity upon cellular internalization. Reprinted with permission from Girard et al., Contrast Media \& Molecular Imaging 2012, 7(4), 411. Copyright 2012 John Wiley and Sons.

Figure 3: Magnetic susceptibility measurements for various fractions of free and cell-internalized IONPs. The susceptibility per unit concentration is independent of IONP local distribution. Reprinted with permission from Girard et al., Contrast Media \& Molecular Imaging 2012, 7(4), 411. Copyright 2012 John Wiley and Sons.

Figure 4: Demonstration of Quantitative Susceptibility Mapping on IONP labeled cells. a) Conventional $\mathrm{T}_{2}$ * weighted magnitude image where IONPs appear as signal voids. b) Background filtered MR phase image: typical magnetic field dipolar pattern is observed around IONP labeled cells. Such a filtered phase map is a prerequisite for susceptibility extraction, or equivalently magnetic moment. c) Magnetic moment map: the image intensity is directly proportional to the total amount of IONP here. See de Rochefort et al., Proc. of ISMRM 2008, 1691, for full description of the acquisition protocol and post-processing, as well as IONP quantification validation.

\section{Financial \& competing interests disclosure}

OMG and RFM were supported in part by the NIH ICMIC P50-CA128346.

The authors have no relevant affiliations or financial involvement with any organization or entity with a financial interest in or financial conflict with the subject matter or materials discussed in the manuscript.

No writing assistance was utilized in the production of this manuscript. 


\section{References}

[1] Lauffer RB. Paramagnetic Metal-Complexes as Water Proton Relaxation Agents for Nmr Imaging - Theory and Design. Chemical Reviews 87(5), 901-927 (1987).

[2] Laurent S, Forge D, Port $M$, et al. Magnetic iron oxide nanoparticles: Synthesis, stabilization, vectorization, physicochemical characterizations, and biological applications. Chemical Reviews 108(6), 2064-2110 (2008).

[3] Girard OM, Du J, Agemy L, et al. Optimization of Iron Oxide Nanoparticle Detection Using Ultrashort Echo Time Pulse Sequences: Comparison of T1, T2*, and Synergistic T1-T2* Contrast Mechanisms. Magn. Reson. Med. 65(6), 1649-1660 (2011).

[4] Yarnykh VL. Actual flip-angle imaging in the pulsed steady state: A method for rapid threedimensional mapping of the transmitted radiofrequency field. Magn. Reson. Med. 57(1), 192200 (2007).

[5] Bowen CV, Zhang XW, Saab G, et al. Application of the static dephasing regime theory to superparamagnetic iron-oxide loaded cells. Magn. Reson. Med. 48(1), 52-61 (2002).

[6] Dahnke H, Schaeffter T. Limits of detection of SPIO at 3.0 T using T-2* relaxometry. Magn. Reson. Med. 53(5), 1202-1206 (2005).

[7] Donahue KM, Weisskoff RM, Burstein D. Water diffusion and exchange as they influence contrast enhancement. Jmri-Journal of Magnetic Resonance Imaging 7(1), 102-110 (1997).

[8] Weisskoff RM, Zuo CS, Boxerman JL, et al. Microscopic Susceptibility Variation and Transverse Relaxation - Theory and Experiment. Magn. Reson. Med. 31(6), 601-610 (1994).

[9] Yablonskiy DA, Haacke EM. Theory of NMR signal behavior in magnetically inhomogeneous tissues: the static dephasing regime. Magn Reson Med 32(6), 749-763 (1994).

[10] Querol M, Bogdanov A. Amplification strategies in MR imaging: Activation and accumulation of sensing contrast agents (SCAs). J. Magn. Reson. Imaging 24(5), 971-982 (2006).

[11] Perez JM, Josephson L, O'Loughlin T, et al. Magnetic relaxation switches capable of sensing molecular interactions. Nature Biotechnology 20(8), 816-820 (2002).

[12] Tu CQ, Osborne EA, Louie AY. Activatable $T$ (1) and $T$ (2) Magnetic Resonance Imaging Contrast Agents. Ann. Biomed. Eng. 39(4), 1335-1348 (2011).

[13] Hoelscher UC, Lother S, Fidler F, et al. Quantification and localization of contrast agents using delta relaxation enhanced magnetic resonance at 1.5 T. MAGMA 25(3), 223-231 (2012).

[14] Terreno E, Crich SG, Belfiore S, et al. Effect of the intracellular localization of a Gd-based imaging probe on the relaxation enhancement of water protons. Magn. Reson. Med. 55(3), 491-497 (2006).

[15] Kok MB, Hak S, Mulder WJ, et al. Cellular compartmentalization of internalized paramagnetic liposomes strongly influences both T1 and T2 relaxivity. Magn Reson Med 61(5), 1022-1032 (2009).

[16] Levy $M$, Wilhelm $C$, Devaud $M$, et al. How cellular processing of superparamagnetic nanoparticles affects their magnetic behavior and NMR relaxivity. Contrast Media Mol Imaging 7(4), 373-383 (2012).

[17] Kuhlpeter R, Dahnke H, Matuszewski L, et al. R2 and R2*mapping for sensing cell-bound superparamagnetic nanoparticles: In vitro and murine in vivo testing. Radiology 245(2), 449457 (2007).

[18] Girard OM, Ramirez R, McCarty S, et al. Toward absolute quantification of iron oxide nanoparticles as well as cell internalized fraction using multiparametric MRI. Contrast Media Mol. Imaging 7(4), 411-417 (2012).

[19] Haacke EM, Xu YB, Cheng YCN, et al. Susceptibility weighted imaging (SWI). Magn. Reson. Med. 52(3), 612-618 (2004).

[20] de Rochefort L, Liu T, Kressler B, et al. Quantitative Susceptibility Map Reconstruction from MR Phase Data Using Bayesian Regularization: Validation and Application to Brain Imaging. Magn. Reson. Med. 63(1), 194-206 (2010). 
[21] Schweser F, Deistung A, Lehr BW, et al. Quantitative imaging of intrinsic magnetic tissue properties using MRI signal phase: An approach to in vivo brain iron metabolism? Neuroimage 54(4), 2789-2807 (2011).

[22] Mills PH, Hitchens TK, Foley LM, et al. Automated detection and characterization of SPIOlabeled cells and capsules using magnetic field perturbations. Magn. Reson. Med. 67(1), 278289 (2012).

[23] Langley J, Liu W, Jordan EK, et al. Quantification of SPIO Nanoparticles in vivo Using the Finite Perturber Method. Magn. Reson. Med. 65(5), 1461-1469 (2011).

[24] Haacke EM, Tang J, Neelavalli J, et al. Susceptibility Mapping as a Means to Visualize Veins and Quantify Oxygen Saturation. J. Magn. Reson. Imaging 32(3), 663-676 (2010).

[25] de Rochefort L, Brown R, Prince MR, et al. Quantitative MR susceptibility mapping using piece-wise constant regularized inversion of the magnetic field. Magn. Reson. Med. 60(4), 1003-1009 (2008). 


\section{Figures}

Figure 1

a) Tumbling rate modulation

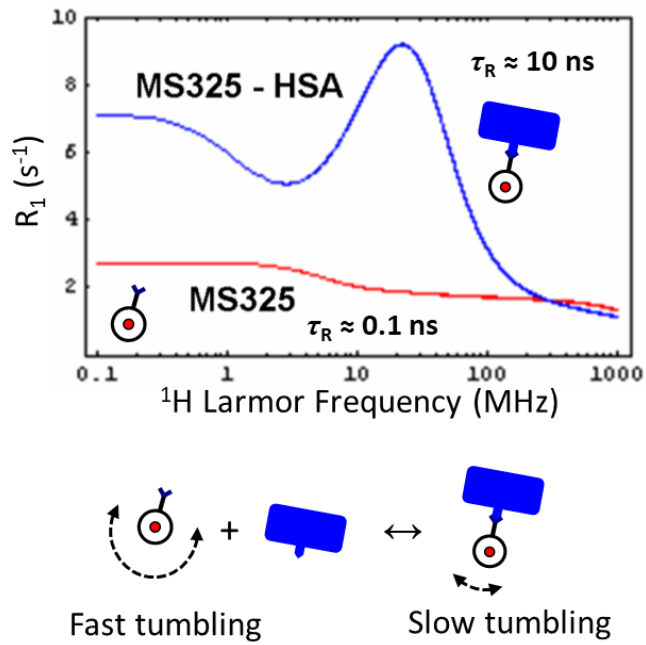

b) Water exchange modulation
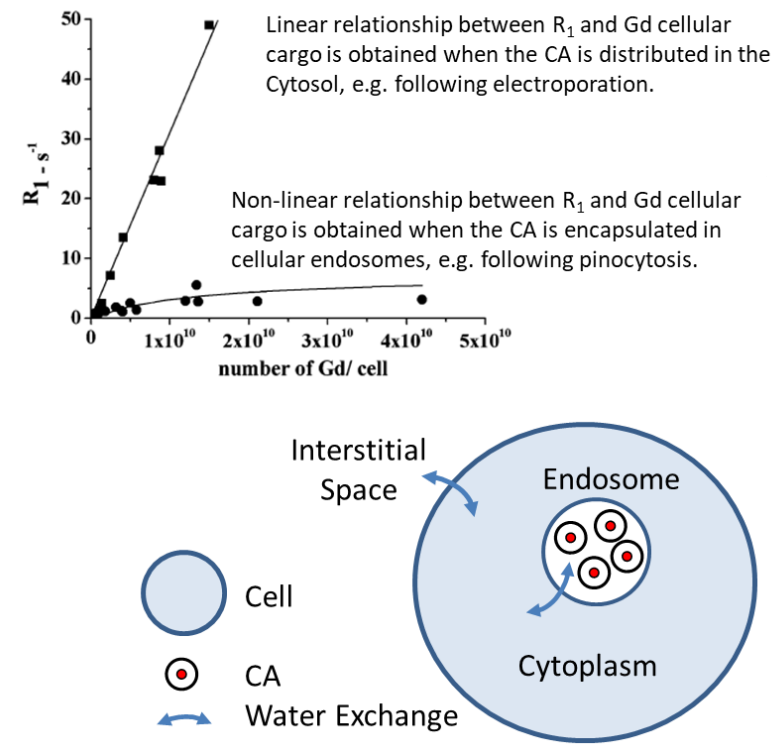
c) IONP clustering modulation

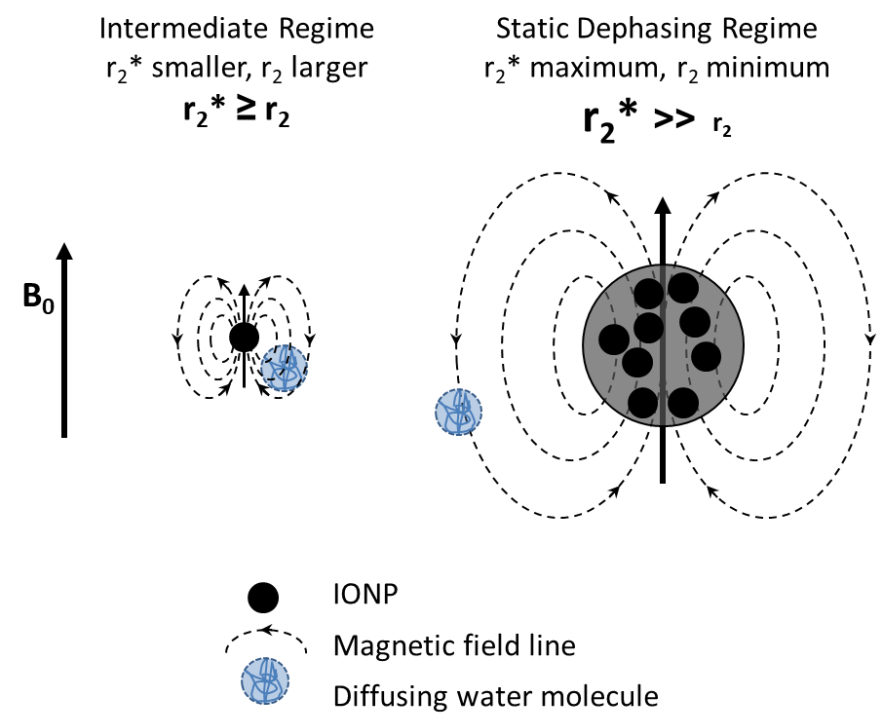

Relaxivity modulation scenarios following receptor binding and cellular uptake. a) Tumbling rate effect following receptor binding for paramagnetic CA. Relaxation dispersion profile for $0.25 \mathrm{mM}$ of MS-325 (Gd) in pure water and in 0.6 mM HSA. See Girard et al. Proc. of ISMRM 2008, 1656, for more information. b) Relaxivity quenching effect following cell internalization and endosomal encapsulation. Such effect is due to the water exchange slowdown through multiple cellular membranes, and hence may affect all kinds of CA. Adapted with permission from Aime et al., Acc. Chem. Res. 2009, 42, 822. Copyright 2009 American Chemical Society. c) Effect of IONP clustering on the $T_{2}$ and $T_{2}{ }^{*}$ contrast regime. $R_{2}{ }^{\prime}=R_{2}{ }^{*}-R_{2}$ is mostly sensitive to IONP clusters such as those encountered into labeled cells. 
Figure 2
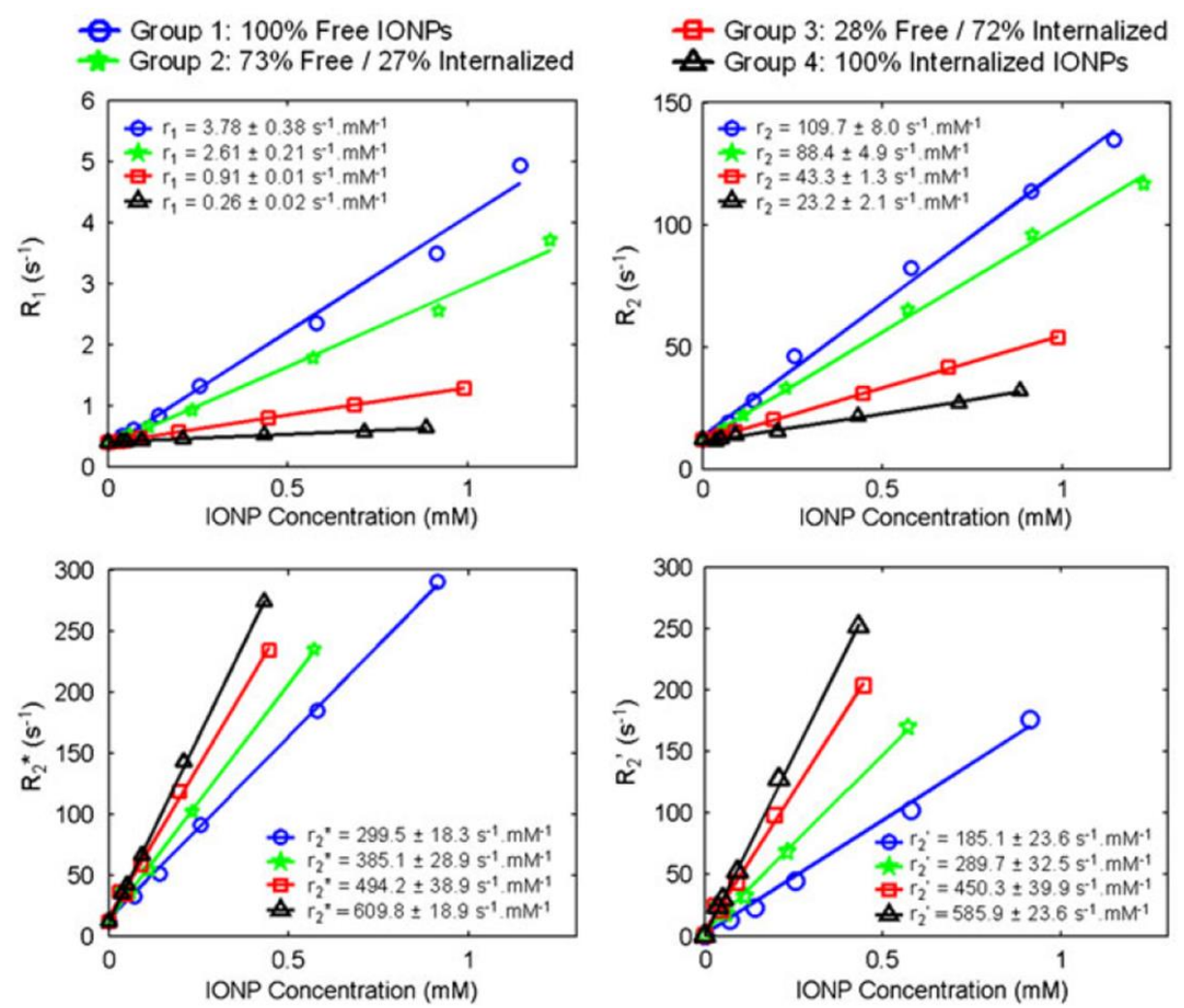

Relaxation rates and corresponding relaxivities for various fractions of free and cell-internalized IONPs. Large relaxivity variations are observed between different groups, evidencing modulation of relaxivity upon cellular internalization. Reprinted with permission from Girard et al., Contrast Media \& Molecular Imaging 2012, 7(4), 411. Copyright 2012 John Wiley and Sons. 
Figure 3

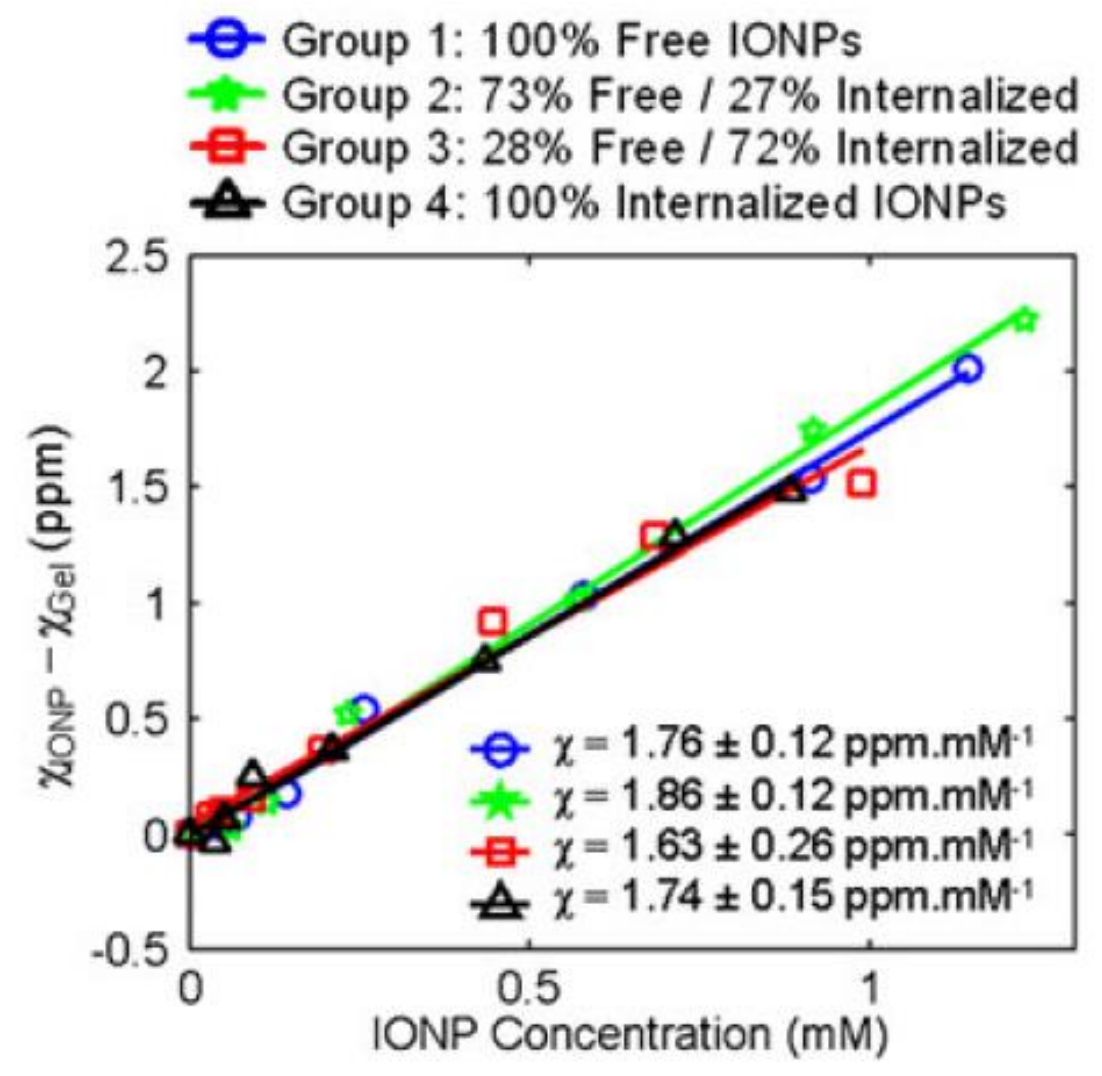

Magnetic susceptibility measurements for various fractions of free and cell-internalized IONPs. The susceptibility per unit concentration is independent of IONP local distribution. Reprinted with permission from Girard et al., Contrast Media \& Molecular Imaging 2012, 7(4), 411. Copyright 2012 John Wiley and Sons. 
Figure 4

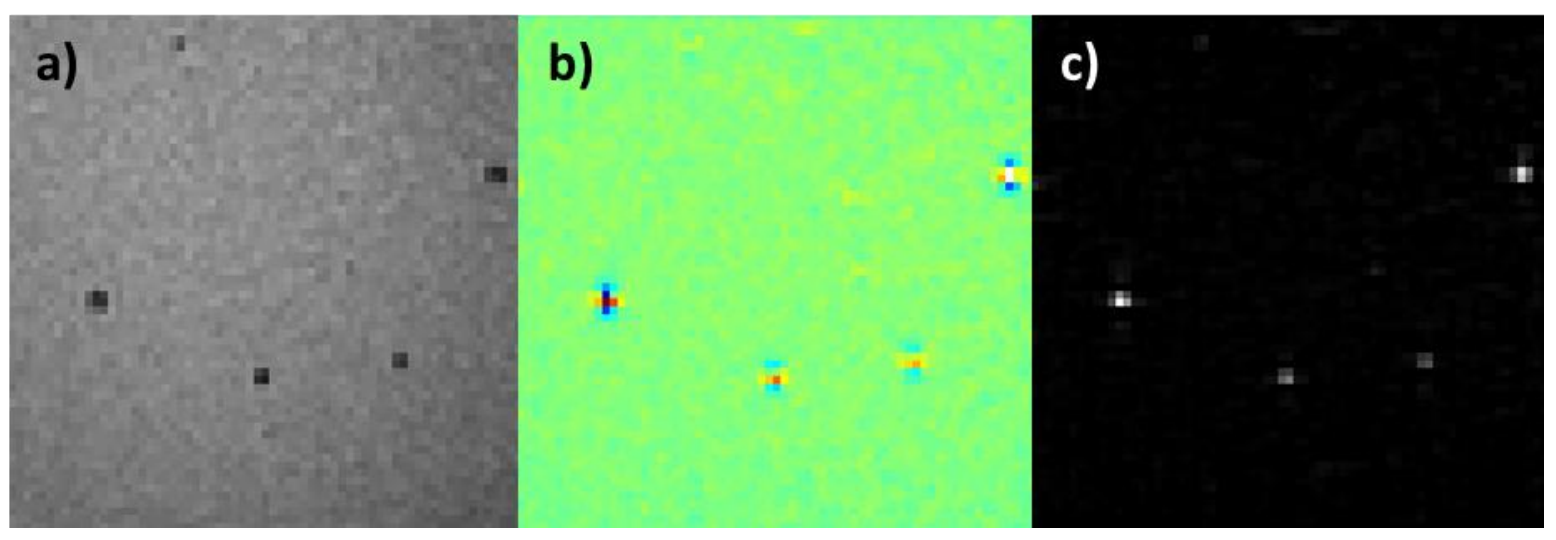

Demonstration of Quantitative Susceptibility Mapping on IONP labeled cells. a) Conventional $\mathrm{T}_{2}{ }^{*}$ weighted magnitude image where IONPs appear as signal voids. b) Background filtered MR phase image: typical magnetic field dipolar pattern is observed around IONP labeled cells. Such a filtered phase map is a prerequisite for susceptibility extraction, or equivalently magnetic moment. c) Magnetic moment map: the image intensity is directly proportional to the total amount of IONP here. See de Rochefort et al., Proc. of ISMRM 2008, 1691, for full description of the acquisition protocol and post-processing, as well as IONP quantification validation. 